Revue internationale P.M.E.

\title{
L'appropriation du système de contrôle de gestion par le propriétaire-dirigeant de petite entreprise : trois étapes en lien avec le concepteur
}

The mental appropriation process by a small business owner-manager of a management control system: three steps in liaison with the designer

\section{La apropiación del sistema de control de gestión por el dirigente-propietario de pequeña empresa: tres etapas en relación con el diseñador}

\section{Odile Bernard}

Volume 32, numéro 1, 2019

URI : https://id.erudit.org/iderudit/1059182ar

DOI : https://doi.org/10.7202/1059182ar

\section{Aller au sommaire du numéro}

Éditeur(s)

Editions EMS - In Quarto SARL

ISSN

0776-5436 (imprimé)

1918-9699 (numérique)

Découvrir la revue

Citer cet article

Bernard, O. (2019). L'appropriation du système de contrôle de gestion par le propriétaire-dirigeant de petite entreprise : trois étapes en lien avec le concepteur. Revue internationale P.M.E., 32(1), 101-125.

https://doi.org/10.7202/1059182ar

\section{Résumé de l'article}

La création d'un système de contrôle de gestion par un concepteur nécessite ultérieurement une appropriation de la part de l'utilisateur. La grille d'analyse, construite à l'issue de la revue de la littérature, permet de repérer l'étape atteinte dans le processus d'appropriation composé de trois phases séquentielles. Une approche qualitative, par entretiens sur site, est réalisée dans quinze petites entreprises du second oeuvre du bâtiment. Le propriétaire-dirigeant est face à l'option de concevoir son système de contrôle de gestion, d'en externaliser la conception auprès de l'expert-comptable, ou de déléguer, en l'occurrence auprès du métreur. L'étude sur le terrain met en avant l'échec récurrent de cette appropriation lorsque le propriétaire-dirigeant n'est pas lui-même le concepteur, échec suivi de l'abandon du contrôle de gestion. Sont en cause, d'une part, la faiblesse de la communication avec l'expert-comptable en termes de formalisation de la vision pour l'avenir de l'entreprise, d'autre part, l'absence de prise en considération par le métreur de la polyvalence de la gestion. Les préconisations portent sur la faculté du propriétaire-dirigeant à transformer ses schèmes de réflexion ; en effet, la divergence entre le travail du concepteur et l'attente de l'utilisateur crée un déséquilibre potentiellement bénéfique à une évolution. 


\title{
L'appropriation du systeme de contrôle de gestion par le propriétaire-dirigeant de petite entreprise : trois étapes en lien avec le concepteur
}

\author{
Odile BERNARD
}

Odile Bernard est docteure en sciences de gestion de l'université Paris-Est Marne-la-Vallée et enseignant-chercheur à l'École de management de Normandie. Une expérience professionnelle, antérieure, en PME du second ouvre du Bâtiment, l'a amenée à étudier les outils et les pratiques de contrôle de gestion en petite entreprise.

École de management de Normandie

Laboratoire Métis

9, rue Claude-Bloch

14052 CAEN CEDEX 4, France

obernard@em-normandie.fr

\section{RÉSUMÉ}

La création d'un système de contrôle de gestion par un concepteur nécessite ultérieurement une appropriation de la part de l'utilisateur. La grille d'analyse, construite à l'issue de la revue de la littérature, permet de repérer l'étape atteinte dans le processus d'appropriation composé de trois phases séquentielles. Une approche qualitative, par entretiens sur site, est réalisée dans quinze petites entreprises du second cuvre du bâtiment. Le propriétaire-dirigeant est face à l'option de concevoir son système de contrôle de gestion, d'en externaliser la conception auprès de l'expert-comptable, ou de déléguer, en l'occurrence auprès du métreur. L'étude sur le terrain met en avant l'échec récurrent de cette appropriation lorsque le propriétaire-dirigeant n'est pas lui-même le concepteur, échec suivi de l'abandon du contrôle de gestion. Sont en cause, d'une part, la faiblesse de la communication avec l'expert-comptable en termes de formalisation de la vision pour l'avenir de l'entreprise, d'autre part, l'absence de prise en considération par le métreur de la polyvalence de la gestion. Les préconisations portent sur la faculté du propriétaire-dirigeant à transformer ses schèmes de réflexion; en effet, la divergence entre le travail du concepteur et l'attente de l'utilisateur crée un déséquilibre potentiellement bénéfique à une évolution.

\section{Mots-CLÉS}

Appropriation, Propriétaire-dirigeant de PE, Concepteur, Systeme de contrôle de gestion 


\title{
The mental appropriation process by a small business owner-manager of a management control system: three steps in liaison with the designer
}

\begin{abstract}
The creation of a Management Control System by a designer will require appropriation by the user later on. The analysis grid proposed, an outcome of a literature review, allows to distinguish the stage reached in the appropriation process, which comprises three sequential phases. A qualitative approach is applied to the case of 15 Small Businesses in the Second Fix Building Sector. The proprietor-manager can either design his/her Management Control System, or externalise this to the Chartered Accountant, or delegate to the quantity surveyor. Field work reveals recurrent failure in the appropriation when the proprietor-manager is not himself/herself the designer, such failure leading to dropping Management Control altogether. The reasons for this are, on the one hand, the poor quality of communication with the Chartered Accountant as regards a formalised vision of the future of the enterprise, and on the other hand, the lack of awareness of the multifarious character of management by the Quantity Surveyor. Recommendations focus on the proprietor-manager's ability to modify his/her patterns of thinking; indeed, the gap between the designer's work and the user's expectations generates an imbalance which has a potentially beneficial contribution to future evolution.
\end{abstract}

KEYWORDS

Appropriation, Small Business Manager, Designer, Management Control System

\section{La apropiación del sistema de control de gestión por el dirigente-propietario de pequeña empresa: tres etapas en relación con el diseñador}

\begin{abstract}
RESUMEN
La creación de un sistema de control de gestión por un diseñador requiere, posteriormente, una apropiación por parte del usuario. La matriz de análisis, procedente del estudio de la literatura académica, permite identificar la etapa alcanzada en el proceso de apropiación. Este último se compone de tres fases secuenciales. Se realiza un enfoque cualitativo, a través de entrevistas in situ, en 15 pequeñas empresas pertenecientes al sector de la construcción. El dirigente-propietario se enfrenta a la opción de elaborar su sistema de control de gestión, externalizarlo a un perito contable o delegarlo a un tasador. El estudio empírico destaca la falla recurrente de la mencionada apropiación cuando el dirigente-propietario no es el diseñador, así tras tal fracaso se abandonará el control de la gestión. Lo que se explica, por un lado, por una insuficiente estrategia de comunicación con el perito contable para formalizar la visión futura de la empresa y, por otro lado, por la falta de consideración del tasador sobre la polivalencia de la gestión. Las recomendaciones se refieren a la capacidad del dirigente-propietario de transformar sus esquemas mentales; de hecho, la discrepancia entre el trabajo del diseñador y las expectativas del usuario crea un desequilibrio que podría favorecer su evolución.
\end{abstract}

Palabras clave

Apropiación, Dirigente de pequeña empresa, Diseñado, Sistema de control de gestión 


\section{INTRODUCTION}

Chaque PME évolue dans un contexte propre et avec des acteurs spécifiques ; aussi, les pratiques de gestion sont-elles soumises aux facteurs de contingence organisationnels, individuels, comportementaux et environnementaux (Dangereux, Chapellier et Villesèque-Dubus, 2017). Le contrôle de gestion en PME est défini par Nobre et Zawadzky (2014) comme un élément fondateur, structurant et à orientation collective. Concernant l'étude de ce contrôle de gestion, au-delà de la dimension technique, c'est l'approche par les relations humaines qui est riche.

Nous parlerons de contrôle de gestion (CdG) pour l'action et de système de contrôle de gestion (SCdG) pour les procédures mobilisées. Un SCdG standardisé n'est pas adapté aux PME, en raison des particularités du contexte des prises de décision et du profil du dirigeant. Dangereux, Chapellier et Villesèque-Dubus (2017) présentent deux écoles concernant le SCdG en PME : soit le SCdG est simple afin de refléter le fonctionnement de la PME, soit le SCdG est spécifiquement adapté à chaque PME en fonction de ses facteurs de contingence. Selon Chapellier, Mohammed et Teller (2013), les dirigeants utilisent principalement des données informelles, des sources externes et non financières; lorsque des données comptables sont utilisées, elles sont "simplifiées et adaptées ", elles servent à identifier les problèmes.

La petite entreprise (PE) se caractérise - au-delà des notions chiffrées - par une forte connotation idiosyncratique de la gestion (Germain, 2006) : centralisation des décisions (Schmitt, Julien et Lachance, 2002), peu de délégation en dehors de la production (Persson-Gehin et Dokaj-Ivanaj, 2002), système d'information informel (Julien, 2005), absence de stratégie définie (Mintzberg, Ahlstrand et Lampel, 2005), motivation des salariés par le mode verbal (Schmitt, Julien et Lachance, 2002), croyances du dirigeant prégnantes dans le choix du système de contrôle de gestion (Davila et Foster, 2005).

Dans le contexte de la PE, la multiplicité des tâches - tâches réalisées bien souvent dans l'urgence - ne permet généralement pas au propriétaire-dirigeant ${ }^{1}$ de dégager du temps pour concevoir un SCdG. Couramment, le p.-dir. délègue ou externalise la conception du SCdG, dont il sera ultérieurement l'utilisateur exclusif. Contrairement à une situation où le p.-dir. construit lui-même son propre SCdG, dans le cadre d'une délégation ou d'une externalisation de la conception du SCdG, l'implémentation nécessite une phase complémentaire : l'appropriation des outils par l'utilisateur (Proulx, 2002 ; Breton et Proulx, 2002 ; Bonneveux et Calmé, 2010). L'appropriation s'effectue suite à l'usage quotidien des outils de CdG par l'utilisateur. En effet, Proulx (2005, p. 10), qui lie le processus d'appropriation à l'arrivée des TIC, le définit comme le "processus d'intériorisation progressive des compétences techniques et cognitives, chez les individus [...] qui manient quotidiennement ces technologies». Au-delà de la maîtrise technique et de la pratique quotidienne, cet auteur étend le processus d'appropriation jusqu'à la possibilité de création autour de ces outils. Ces outils entrent alors dans la configuration des outils construits par l'acteur, selon Solle et Rouby (2003), afin d'être adaptés à une situation très précise. Ces outils « maison », personnalisés par l'acteur

1 «Propriétaire-dirigeant » est désormais remplacé par «p.-dir. », afin d'alléger la lecture. 
correspondent à une évolution, voire à un détournement des outils initiaux, pour produire les conséquences souhaitées.

Sans appropriation par le p.-dir., le travail du concepteur risque de ne pas être mis en œuvre, d'être abandonné. Or, l'appropriation du SCdG est soumise à une obligation de réussite puisqu'elle permet de répondre au besoin de formalisation du CdG, détecté par le p.-dir. En effet, en PE indépendante, ce contrôle n'est pas imposé, mais souhaité par le p.-dir. qui en attend une possible amélioration des performances (DeLone et McLean, 2003 ; Bourguignon, 2004) ; de plus, "la prise de décision est fortement liée à la capacité du p.-dir. à traiter et exploiter l'information disponible» (Barlette et Jaouen, 2015, p. 631).

Afin d'approcher le processus d'appropriation par le p.-dir., l'opportunité a été saisie d'étudier les deux situations de délégation et d'externalisation de la conception du SCdG en PE similaires, d'un secteur d'activité précis - le secteur de fabrication de mobilier d'agencement sur mesure. L'objectif est de comprendre l'incidence du profil du concepteur du SCdG - au regard de sa fonction et de sa formation - sur le processus d'appropriation par l'utilisateur, en l'occurrence le p.-dir. Est-il possible d'identifier et d'expliquer le lien entre le choix du concepteur du SCdG - choix du concepteur entre externalisation et délégation - et l'éventuel échec du processus d'appropriation par le p.-dir.?

L'intérêt de cette recherche est à la fois théorique, car elle permet de mieux comprendre un type de comportement du p.-dir. dans le cadre de l'amélioration de la gestion, et managérial puisqu'elle conduit à des préconisations d'ordre comportemental.

Trois groupes sont étudiés : des p.-dir. qui ont délégué la conception du SCdG, des p.-dir. qui ont externalisé cette conception et ceux qui ont conçu eux-mêmes le SCdG (constituant en quelque sorte un groupe témoin). L'opportunité d'un terrain offrant les trois situations est rare : c'est la présence d'un métreur dans le secteur de la fabrication de mobilier d'agencement sur mesure qui procure sa richesse à ce terrain. L'étude conjointe de la délégation et de l'externalisation de la conception du SCdG permet un apport supplémentaire et atypique aux travaux existants sur la relation des p.-dir. au conseil, largement étudiée dans la littérature. Par exemple, selon Bennett et Robson (1999), l'expert-comptable est la première source de conseil professionnel pour les p.-dir., et plus ce dernier possède la volonté de développer l'entreprise, plus il sera réceptif aux conseils. L'étude actuelle ne concerne pas le processus de choix du concepteur, présent dans les travaux de Cullière (2006). Ce sont les conséquences du choix du concepteur du SCdG sur l'appropriation ultérieure du SCdG, par le p.dir., qui sont ici étudiées.

Dans une première partie, l'étude de la littérature montre la compatibilité des outils de gestion avec la notion d'appropriation, mais aussi identifie les freins d'ordre comportemental inhérents à ce cheminement vers l'appropriation. La synthèse des travaux des auteurs, au sujet du processus d'appropriation, permet l'identification de trois étapes séquentielles et la construction d'une grille de lecture du processus d'appropriation du SCdG par le p.-dir. Dans une deuxième partie, une étude effectuée auprès de quinze PE du secteur de l'agencement sur mesure permet de tester cette grille de lecture et de repérer l'étape à laquelle le processus d'appropriation s'est arrêté par abandon du SCdG proposé. Enfin, une troisième partie - la confrontation de l'étude de terrain à la revue de littérature - autorise à identifier les facteurs susceptibles de faire échouer le processus d'appropriation. 


\section{LAPPROPRIATION DES OUTILS DE GESTION DANS LA LITTÉRATURE}

L'objet de l'appropriation, concernant cette étude, est l'outil de CdG. La littérature porte principalement sur l'outil de gestion, dans lequel est inclus l'outil de CdG. Moisdon (1997, p. 7) définit l'outil de gestion comme « toute formalisation de l'activité organisée, [...] tout schéma de raisonnement reliant de façon formelle un certain nombre de variables issues de l'organisation, et destinées à instruire les divers actes de la gestion $»$. Si les caractéristiques des outils de gestion présentent une potentielle compatibilité avec un processus d'appropriation, de nombreuses conditions comportementales sont, cependant, à considérer.

\subsection{Les caractéristiques des outils de gestion en concordance avec l'appropriation}

Transposables et porteurs de connaissances des mécanismes du fonctionnement de l'entreprise, les outils de gestion favorisent la réflexion. Cependant, les acteurs introduisent des biais comportementaux qui risquent de freiner le processus d'appropriation.

\subsubsection{DE L'AMÉLIORATION DE LA CONNAISSANCE DU FONCTIONNEMENT DE L'ENTREPRISE VERS L'ACTION}

L'outil de gestion est un objet fabriqué (Vaujany (de), 2005) dans un objectif déterminé au préalable et précis, il n'existe qu'à travers l'usage qui en est fait et présente ainsi un caractère instrumental. Même si l'outil apparaît formalisé, l'absence de lien avec le contexte de son utilisation fait qu'il est transposable (David, 1996). En effet, l'outil se transforme suite à l'appropriation par l'utilisateur et présente alors son caractère contextuel.

En tant qu'objet de transmission des connaissances du concepteur vers l'utilisateur (Brasseur et Buisson, 2008), l'outil représente la confrontation du travail du concepteur avec les attentes de l'utilisateur (Vaujany (de), 2005). En apprenant à mettre en œuvre un outil de gestion, l'utilisateur assimile les savoirs du concepteur ; néanmoins il espère aussi y retrouver le reflet de ses propres pratiques et de ses attentes. Le choix du concepteur risque donc fortement d'avoir une incidence sur la possibilité d'appropriation, puisque ses connaissances vont interférer avec les décisions prises par le p.-dir.

Le processus d'appropriation d'un outil aide aussi l'acteur à réfléchir sur le fonctionnement des mécanismes instrumentés. En effet, le cheminement de l'appropriation est défini par David (1996, p. 17) comme « un état ou processus particulier de transformation réciproque de l'outil par les acteurs et des acteurs par l'outil », et Mallet (2006, p. 4) évoque une "construction réciproque de l'individu et de l'outil ». Selon Nieto-Bru (2009), sur le plan individuel, le processus d'appropriation correspond à des modifications de connaissances et/ou de comportement induites par l'apprentissage : révision de son approche du fonctionnement de l'organisation, de sa perception des outils (Grimand, 2012). Autrement dit, la connaissance produite trouve un écho auprès des problématiques quotidiennes. L'interprétation des 
résultats fournis par les outils permet l'amélioration de la connaissance du fonctionnement des mécanismes de l'entreprise, elle l'instrumentalise.

L'objectif de la phase d'appropriation est donc bien de conduire le p.-dir. à prendre des décisions et à les activer, puisque l'actionnabilité des connaissances (Charreire, 2002) est liée à la pertinence des outils au regard du contexte afin de rendre possible la mise en acte des prises de décision.

\subsubsection{LES RISQUES CONCERNANT L'APPROPRIATION INHÉRENTS À LA RELATION ENTRE LES ACTEURS}

"Les outils de gestion ne se déploient jamais sur un terrain vierge, mais doivent composer avec des logiques locales, des territoires institués... " (Bessire, Brillet, Grimand et Meric, 2012, p. 54). De ce fait, il est indispensable d'adapter la " philosophie gestionnaire " (David, 1996) du nouvel outil aux caractéristiques de l'organisation. Les travaux de Mole (2002) mettent en avant le respect de la spécificité du secteur d'activité de l'entreprise (a), de la culture d'entreprise (b) et surtout le besoin d'une relation de proximité et d'échanges entre le concepteur et l'utilisateur (c), afin de favoriser le processus d'appropriation :

a) L'implémentation d'un outil de gestion comporte une action structurante sur l'entreprise (Moisdon, 1997) ; de plus, les p.-dir. de PE sont convaincus de l'unicité de leur mode de gestion (Dalley et Hamilton, 2000). Le concepteur ${ }^{2}$ est ainsi contraint de saisir «l'entreprise à la fois dans sa structure, dans sa dynamique et dans ses objectifs » (Cullière, 2006, p. 180) et de prendre en considération l'identité de l'entreprise. Or, les p.-dir. doutent de la bonne compréhension du contexte de l'entreprise par les conseillers (Dyer et Ross, 2007). La proposition finale du conseiller est trop souvent qualifiée de générique et non de spécifique (Dalley et Hamilton, 2000 ; Mole, 2002 ; Bennett et Robson, 2005) ; or, Mole (2002) démontre que c'est le niveau de spécialisation qui donne sa qualité au conseil. Ainsi, le biais à craindre est que le concepteur incite le p.-dir. à adopter sa façon d'envisager le problème à résoudre, à épouser son mode de raisonnement théorique (Dyer et Ross, 2007). Gibb (2000) met en garde contre la tendance des conseillers à favoriser leurs centres d'intérêt dans leurs préconisations. Le risque est que le conseiller donne des priorités trop guidées par sa propre expertise et influence fortement l'orientation du SCdG.

b) Selon Mole (2002), la pérennité des outils implémentés dépend de la compatibilité de la culture du concepteur et de celle de l'utilisateur, ainsi que de leurs valeurs respectives. En effet, il existe entre les organisations des divergences au niveau des croyances, des valeurs, des relations entre ses acteurs, de la culture. Si le savoir transmis ne correspond pas au contexte culturel de l'entreprise, la tentative de transfert de connaissances échoue (Moles, 2002), car le p.-dir. ne retrouve pas la propre identité de l'entreprise (Dyer et Ross, 2007).

c) La relation d'externalisation ou de délégation est trop souvent sous-tendue par des hypothèses de la part du concepteur concernant le résultat à obtenir et les attentes de l'utilisateur (Stumpf et Longman, 2000) ; la principale critique porte sur ce risque de nonadéquation (Marriott et Marriott, 2000). Un riche échange d'informations de la part des

2 Les études appréhendées concernent principalement le conseil (par un consultant), et sont ici transposées à la conception d'un SCdG. Aussi, le concepteur est-il assimilé à un conseiller. 
deux parties (Bennett et Robson, 2005) est nécessaire pour favoriser la qualité du SCdG. En effet, l'asymétrie des connaissances renforce le besoin de synergie (Wang et Chen, 2006) : pour l'efficacité du résultat obtenu, les deux acteurs doivent se comprendre parfaitement (Gable et Chin, 2001). L'idée de compréhension est liée à celle d'expression des attentes du p.-dir., tant sur le long terme que concernant les problèmes quotidiens. Une éventuelle divergence d'idées est sans conséquence à condition que la communication entre les deux parties soit suffisante et associée à des confrontations d'opinions enrichissantes (Dyer et Ross, 2007). De plus, des facteurs facilitateurs peuvent être identifiés (Cullière, 2006) : la qualité du contact, l'empathie, la pédagogie, dont fait preuve le concepteur.

Enfin, Gervais (2005) considère que le degré de contextualisation d'un SCdG est souvent insuffisant, car l'acteur responsable de la conception doit aussi apporter sa créativité, l'imagination de l'acteur restant toujours nécessaire lorsqu'il s'agit de faire face à une situation totalement nouvelle.

Ainsi, si les caractéristiques des outils de gestion les rendent propices à l'appropriation, le contexte comportemental tient une large place dans la réussite.

\subsection{Un processus d'appropriation du système de contrôle de gestion en trois étapes séquentielles}

La synthèse de la littérature permet de distinguer trois étapes séquentielles du processus d'appropriation, qui sont récurrentes dans les définitions des auteurs (Proulx, 2002; Breton et Proulx, 2002 ; Vaujany (de), 2005 ; Bonneveux, Calmé et Soparnot, 2016). Ce processus est décrit par Bonneveux et Calmé (2010, p. 4) comme comportant une première phase d'accès, une deuxième d'utilisation puis une troisième de transformation de l'objet. Plus précisément, ces auteurs définissent un « apprentissage en produisant " (adoption), un " apprentissage en utilisant " (" assimilation ", au sens de Piaget, 1973) et un "apprentissage en interagissant " ("accommodation », au sens de Piaget, 1973). Ainsi, l'apport théorique de cette revue de la littérature sera la réalisation d'une grille de lecture concernant les étapes du processus d'appropriation ${ }^{3}$.

\subsubsection{Première ÉtAPE : MAîtTrise COgNitive des OUTILS PAR L'UTILISATEUR}

La première étape vers l'appropriation considère le substrat technique du dispositif (David, 1996) ; il s'agit de la maîtrise cognitive, ou acquisition des compétences requises pour l'utilisation de l'objet technique. Cette étape demande au p.-dir. de comprendre le mécanisme de fonctionnement des outils de CdG proposés et surtout de savoir interpréter les résultats obtenus. En effet, le premier stade de l'appropriation est constitué par le « mécanisme par lequel l'individu va acquérir des connaissances sur l'outil » (Massard, 2009, p. 2). Concrètement,

3 Le nom de chacune des trois étapes du processus d'appropriation, pour la grille de lecture, émanera des dénominations selon Bonneveux et Calmé (2010), soit : 1) adoption, 2) utilisation contextualisée et 3) interaction. 
cette étape se traduit par une période de test des outils avec des données chiffrées concernant une période révolue ou en cours.

La maîtrise technique conditionne le passage à la deuxième étape de l'appropriation, qui est la capacité à donner un sens à l'ensemble du dispositif technique (Massard, 2009 ; Bonneveux et Calmé, 2010), c'est-à-dire à le contextualiser de par son utilisation en situation.

\subsubsection{DEUXIÈME ÉTAPE : UTILISATION SOUS-TENDANT LA MISE EN ADÉQUATION AVEC LE CONTEXTE ET LES ATTENTES DU PROPRIÉTAIRE-DIRIGEANT}

La deuxième étape est l'acceptation des outils dans le quotidien de l'acteur et leur intégration dans les routines de travail. Il s'agit d'une phase de repérage de la cohérence entre le SCdG proposé et les attentes de l'utilisateur. Elle consiste en une confrontation des outils avec les souhaits du p.-dir. en termes de suivi, d'aide à la prise de décision et éventuellement de pilotage. Lors de la mise en œuvre du CdG, le p.-dir. s'attend à obtenir des réponses concrètes à ses interrogations préalables, il escompte que le nouvel outil sera en adéquation avec son approche du fonctionnement de l'entreprise, sa politique d'entreprise, sa vision de l'avenir, ses valeurs, ceci dans le contexte économique et environnemental propre à chaque entreprise. Durant cette étape, le p.-dir. teste la contextualisation de la proposition de SCdG et tente de l'adapter à une destination précise (Massard, 2009). Cette étape consiste à réduire l'écart entre l'outil et l'organisation, ce que David (1996) nomme la "distance "; Vaujany (de) (2005) propose de viser la « digestion de l'outil», son « internalisation ».

Lorsque l'utilisateur du SCdG cherche à rendre compatibles ses attentes, en termes de mise sous contrôle, ainsi que sa culture d'entreprise et sa perception de l'environnement avec les outils proposés par le concepteur, ses croyances au niveau des besoins de l'entreprise en CdG restent inchangées. Il s'agit de la phase que Piaget (1973) nomme " assimilation » : les schèmes cognitifs ne sont pas remis en question. La gestion de l'entreprise est perfectionnée, ouverte au contrôle et par conséquent aux prises de décisions justifiées, mais l'objectif poursuivi est simplement mieux géré, il n'est ni influencé ni modifié. L'assimilation consiste donc en l'intégration de nouveaux outils dans la structure mentale de l'utilisateur du SCdG.

Les outils qui ne répondent pas à cette mise en correspondance avec les certitudes de l'utilisateur sont abandonnés, on constate alors leur rejet par l'utilisateur (Abdallah, 2007).

La deuxième étape du processus d'appropriation consiste donc en l'activité « d'adaptation et de mise en adéquation de l'outil avec une utilisation déterminée » (Perez, Chalayer-Rouchon et Teyssier, 2005, p. 2). L'utilisateur est alors prêt pour aborder la troisième étape du processus d'appropriation : la créativité par l'interaction avec l'outil.

\subsubsection{TROISIÈME ÉTAPE : INTERACTION, RÉINTERPRÉTATION ET CRÉATIVITÉ AUTOUR DES OUTILS}

Les nouveaux outils proposés constituent des stimuli qui créent une interaction bénéfique entre l'acteur et son environnement. La perturbation cognitive, entraînée par la deuxième étape, a éventuellement permis de déclencher une instabilité favorable à l'évolution des représentations de l'utilisateur (Grimand, 2006), elle participe à amplifier sa capacité d'analyse 
et à améliorer ses pratiques de gestion (Charreire, 2002). La phase précédente, d'assimilation, a potentiellement permis la création d'un conflit entre les attentes de l'acteur et les propositions du concepteur, conflit comparable à un déséquilibre. Ce déséquilibre est résorbé par une nouvelle situation ajustée au niveau des schèmes cognitifs ; elle est appelée « rééquilibration» (Piaget, 1973).

La troisième étape correspond donc à la démarche la plus avancée dans le processus d’appropriation puisqu'elle amène l'utilisateur du SCdG à réviser son approche du CdG, généralement à l'élargir, de manière à intégrer les possibilités suggérées par les outils. Il est invité à réviser son mode de gestion, voire sa stratégie, ou du moins sa ligne directrice. Le comportement, au regard du CdG, se trouve modifié, les croyances et les convictions sont réévaluées à la lumière des possibilités offertes par le SCdG élaboré par le concepteur. L'appropriation du SCdG a généré l'accommodation - au sens de Piaget (1973) - des schèmes cognitifs de l'utilisateur. L'appropriation à ce stade a encouragé la remise en question des méthodes souhaitées ou mise en œuvre préalablement. La modification des schèmes cognitifs autorise l'incorporation des outils dans de nouvelles configurations souvent plus performantes. Le comportement et la réflexion ont évolué grâce à la confrontation avec le travail d'une tierce personne : le concepteur du SCdG. Cette étape peut entraîner une création de valeur (Massard, 2009) grâce à de nouvelles pratiques.

À l'inverse, l'échec de l'accommodation est suivi d'un désintérêt et d'une situation de statu quo au regard du SCdG proposé.

La troisième étape amène l'utilisateur à faire preuve de créativité, créativité dynamique qui traduit l'intérêt développé par l'acteur pour l'objet à améliorer (Hussenot, 2008). Cette phase constitue une période de questionnement pendant laquelle l'acteur imprime à l'outil son style (Grimand, 2006). Proulx (2002), Solle et Rouby (2003), Vaujany (de) (2005) et Massard (2009) montrent les possibilités de réinterprétation et d'instrumentation, voire de détournements de l'outil. Bessire et al. (2012) affirment que des usages autres que ceux prévus initialement sont cohérents, qu'il ne s'agit pas d'une erreur ou d'une transgression.

Cette troisième étape autorise donc à affirmer que l'appropriation constitue un processus non limité dans le temps.

\subsubsection{GRILLE DE LECTURE DES ÉTAPES SÉQUENTIELLES DU PROCESSUS D'APPROPRIATION}

Le fruit du croisement des différentes littératures concernant le processus d'appropriation est la construction d'une grille de lecture. Produit de l'analyse synthétique des travaux existants, cette grille de lecture représente un outil de repérage de l'étape lors de laquelle a lieu l'arrêt du processus d'appropriation, associé aux notions d'assimilation et d'accommodation concernant l'apprentissage. Les trois étapes identifiées permettront une mise en perspective des résultats concernant le processus d'appropriation dans le contexte de la PE, résultats qui seront lus au regard des risques identifiés, inhérents à la relation entre les acteurs lors des situations d'externalisation ou de délégation de la conception du SCdG. 


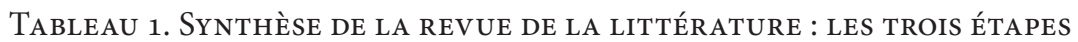
SÉQUENTIELLES DU PROCESSUS D'APPROPRIATION DU SYSTÈME DE CONTRÔLE DE GESTION PAR LE PROPRIÉTAIRE-DIRIGEANT

\begin{tabular}{|c|c|c|c|}
\hline $\begin{array}{l}\text { Étapes } \\
\text { séquentielles }\end{array}$ & $\begin{array}{l}\text { Caractéristiques de } \\
\text { chaque étape }\end{array}$ & $\begin{array}{l}\text { Risques spécifiques } \\
\text { pour le p.-dir. }\end{array}$ & $\begin{array}{l}\text { Théorie associée au } \\
\text { processus }\end{array}$ \\
\hline $\begin{array}{l}\text { Étape } 1 \\
\text { Adoption }\end{array}$ & $\begin{array}{l}\text { Maîtrise cognitive du } \\
\text { mécanisme des outils et } \\
\text { de l'interprétation des } \\
\text { résultats obtenus }\end{array}$ & $\begin{array}{l}\text { Risque du non-repérage } \\
\text { des modifications à } \\
\text { apporter pour répondre à } \\
\text { ses attentes } \\
\text { Focalisation sur l'absence } \\
\text { de pertinence, au regard } \\
\text { du p.-dir., des objets mis } \\
\text { sous contrôle }\end{array}$ & $\begin{array}{l}\text { Rencontre de l'acteur et } \\
\text { de l'outil }\end{array}$ \\
\hline $\begin{array}{l}\text { Étape } 2 \\
\text { Utilisation } \\
\text { contextualisée }\end{array}$ & $\begin{array}{l}\text { Mise en adéquation des } \\
\text { outils avec les attentes } \\
\text { et les objectifs du p.- } \\
\text { dir., avec le contexte de } \\
\text { l'entreprise et avec la } \\
\text { culture et les valeurs de } \\
\text { l'entreprise }\end{array}$ & $\begin{array}{l}\text { Risque de non-intégration } \\
\text { des outils dans les } \\
\text { routines de travail et les } \\
\text { schémas de réflexion } \\
\text { Concentration sur les } \\
\text { divergences entre les } \\
\text { possibilités du SCdG } \\
\text { proposé et le contexte } \\
\text { perçu par le p.-dir. } \\
\text { Tensions autour } \\
\text { du manque de } \\
\text { correspondance avec ses } \\
\text { attentes } \\
\text { Élimination de certains } \\
\text { outils voire abandon total }\end{array}$ & $\begin{array}{l}\text { Assimilation (Piaget, } \\
\text { 1973) Comportement } \\
\text { modifié, mais } \\
\text { transformation ni des } \\
\text { valeurs ni des croyances }\end{array}$ \\
\hline $\begin{array}{l}\text { Étape } 3 \\
\text { Interaction }\end{array}$ & $\begin{array}{l}\text { Réinterprétation et } \\
\text { création autour et à partir } \\
\text { des outils }\end{array}$ & $\begin{array}{l}\text { Fort risque d'absence de } \\
\text { création ou d'initiatives } \\
\text { autour des outils } \\
\text { Enlisement dans ses } \\
\text { modèles de réflexion sans } \\
\text { écho avec les outils }\end{array}$ & $\begin{array}{l}\text { Accommodation } \\
\text { (Piaget, 1973) Valeurs et } \\
\text { croyances modifiées }\end{array}$ \\
\hline
\end{tabular}

La grille de lecture, constituée des trois étapes séquentielles, sera soumise à l'épreuve des faits : elle va autoriser à identifier la ou les étapes qui posent problème, pour chacune des situations d'externalisation ou de délégation, dans l'avancement du processus d'appropriation. L'étude qualitative, par l'analyse des discours des p.-dir., permettra d'en comprendre les raisons. 


\section{PARTIE EMPIRIQUE : ÉTUDES DE CAS AUPRĖS DE PROPRIÉTAIRES-DIRIGEANTS DE PETITE ENTREPRISE}

La méthodologie qualitative - retenue afin de comprendre les raisons profondes des échecs à la mise en place du SCdG - a orienté cette partie empirique vers des études de cas. La technique de collecte de données choisie est celle de visites en PE et d'entretiens semi-directifs avec les p.-dir. Elle est suivie de l'étude des discours avec codage.

\subsection{Présentation du contexte et des entreprises}

Quinze études de cas sont effectuées auprès de p.-dir. de PE du secteur de fabrication de mobilier d'agencement sur mesure. Le choix de ce secteur d'activité est lié tant à l'expérience professionnelle antérieure de l'auteur qu'à la spécificité de la présence d'un métreur dans chacune de ces PE.

\subsubsection{LES CRITÈRES DE SÉLECTION DES ENTREPRISES}

La sélection des entreprises permet de répondre aux contraintes liées à notre problématique. Entreprise indépendante qui ne se voit pas imposer un SCdG; taille de la PE où le p.-dir. gère lui-même le $\mathrm{CdG}$; entreprise qui a dépassé la période de démarrage et où les besoins en CdG sont stabilisés; entreprise évoluant sur un marché régional de la fabrication sur mesure (effet de proximité des clients, donc concurrence réduite) ; p.-dir. de formation technique et en deuxième partie de carrière (études à l'époque où ce type de formation ne comportait pas d'enseignements en gestion, leurs compétences ont donc été acquises en entreprise).

Une première sélection, par simple rendez-vous téléphonique, permet d'éliminer les entreprises en début de réflexion autour de l'implémentation d'un SCdG, d'identifier les situations d'externalisation et de délégation de la conception du SCdG et de ne conserver que trois p.-dir. autonomes au regard de la conception du SCdG. Ce petit échantillon de référence permet de mieux appréhender les similitudes et les différences de ressenti entre les trois groupes de p.-dir. : ayant délégué, ayant externalisé ou autonomes.

Dans ces entreprises de production sur mesure ou " à la demande ", le SCdG quotidien concerne presque exclusivement l'analyse des informations issues des ateliers. Les différentes étapes de la production donnent lieu à des calculs d'écart entre temps prévu et temps réalisé. En effet, la principale difficulté d'un chiffrage pour une fabrication sur mesure concerne le temps alloué aux opérations, bien plus que la valorisation des matériaux mis en œuvre. Audelà de la vérification de la rentabilité en termes de temps passé, c'est l'apprentissage perpétuel de la décision du temps à mobiliser qui intéresse ces entreprises. De plus, chaque p.-dir. a construit des attentes en termes de tableaux de bord, généralement plus des « repères » que des analyses. On pourrait résumer ces outils par «si... alors » pour la prise de décision. Il existe aussi des rappels de dates-clés, de démarches à effectuer et d'événements à gérer. 


\subsubsection{LES OPPORTUNITÉS À DISPOSITION DU PROPRIÉTAIRE-DIRIGEANT POUR LA CONCEPTION DU SYSTÈME DE CONTRÔLE DE GESTION}

Le p.-dir. de PE souhaite effectuer lui-même le contrôle de gestion opérationnel, comme tactique, mais le manque de temps et des connaissances avant tout techniques le contraignent très souvent à solliciter un concepteur pour son SCdG.

Lors du choix de déléguer la conception du SCdG, dans ces entreprises de fabrication de mobilier d'agencement sur mesure, la présence d'un métreur constitue un apport de compétences assimilables à celles en CdG, spécifiques à ce secteur d'activité au regard de notre problématique. Généralement de formation technique ou technico-commerciale, à l'aise avec les chiffrages, concepteur des plans de fabrication, acheteur des matériaux et négociateur auprès des fournisseurs, interface entre les salariés en atelier et le p.-dir., ainsi qu'entre les clients et le p.-dir., ce bras droit du p.-dir. connaît le fonctionnement de l'entreprise dans tous ces rouages. Il constitue un acteur privilégié pour la délégation de la conception du SCdG.

Lorsque le choix se porte sur l'externalisation, de manière non spécifique à ce secteur d'activité, le p.-dir. se tourne très généralement vers l'expert-comptable. Acteur aux connaissances en gestion financière reconnues, il évolue dans de très nombreux secteurs d'activité et accepte généralement un rôle de conseil.

\subsection{Processus de collecte et d'analyse des données}

Dans l'objectif d'utiliser de multiples sources d'information pour les études de cas (Yin, 1989), une journée complète de visite est organisée dans chacune des quinze entreprises, à des fins d'entretiens avec le p.-dir. et d'observation simple, par exemple concernant les fichiers Excel. L'intérêt est de travailler sur les témoignages des p.-dir. concernant les éventuels freins à l'appropriation rencontrés. Les discours, selon Blanchet et Gotman (2005), présentent deux volets : discours modaux pour tenter de traduire l'état psychologique du locuteur, et discours référentiels pour décrire l'état des choses. Le guide d'entretien a donc été construit à l'aide des indicateurs déterminés par la revue de la littérature et par le cadre contextuel. Ce guide permet avant tout d'éviter le poids de l'interprétation. Les données recueillies sont ainsi composées de récits d'événements, d'expressions du ressenti des p.-dir. et de justifications de la situation actuelle.

La pratique d'une triangulation est effectuée, suivant Grimand et Bachelard (2005), grâce à la comparaison entre les documents observés et les discours tenus. Ainsi, les observations pratiquées portent sur le repérage des pratiques managériales et sur les témoignages concernant les relations entre les p.-dir. et le concepteur du SCdG. L'étude documentaire s'effectue donc par la consultation des méthodes de travail, mais aussi par la prise de connaissance des outils formalisés comme informels : calculs, simulations, comparaisons, tableaux de bord, budgets, etc.

À l'issue du recueil des données et de la transcription des entretiens, le codage permet d'organiser les données par thème, en regroupant toutes les parties de discours qui abordent une idée identique. L'objectif est que ce découpage soit stable d'un entretien à l'autre et ne comporte pas de notion d'interprétation. Un premier codage avait émergé de la revue de la littérature, principalement autour des thèmes : objectifs attendus du SCdG, concepteur 
retenu, quel niveau de communication avec le concepteur, avantages et limites du SCdG obtenu, mais à la suite des entretiens, le codage prédéterminé a été revu avec une partie de codage émergent.

À l'issue du codage et des regroupements, l'analyse des données est effectuée. Elle permet d'évaluer la phase d'appropriation du SCdG par le p.-dir., en fonction de la grille comportant trois étapes, mais surtout, l'analyse éclaire sur les raisons de l'éventuel échec de l'appropriation, en fonction des situations d'externalisation et de délégation.

\section{RÉSULTATS : ANALYSE ET COMMENTAIRES}

L'objectif est de déterminer - en fonction du concepteur - l'étape à laquelle le processus d'appropriation du SCdG s'est arrêté. L'analyse des discours, à la lumière de la revue de la littérature, permet de comprendre les raisons des obstacles ayant empêché le passage à l'étape suivante.

\subsection{Avancement du processus d'appropriation du système de contrôle de gestion par le propriétaire-dirigeant}

L’enquête, réalisée auprès de quinze p.-dir. de PE du secteur de l'agencement d'intérieur, permet la construction d'un tableau de synthèse des résultats (Tableau 2). Il croise la situation de délégation, d'externalisation ou d'autonomie ${ }^{4}$ de la conception du SCdG, et met en avant l'étape à laquelle s'est arrêté le processus d'appropriation par le p.-dir. - en fonction de la grille d'analyse issue de la revue de la littérature. Dans les situations d'arrêt du processus d'appropriation, le p.-dir. s'est désintéressé du SCdG proposé par le concepteur. Il convient donc de qualifier d'échec la tentative d'implémentation d'un SCdG, échec d'autant plus dommageable qu'à l'unanimité les p.-dir. n’envisagent pas de réitérer l'expérience.

TABleaU 2. CRoIsement ENTRE LE TyPe DE CONCEPTION DU SYSTÈme DE CONTRÔLE DE GESTION ET L'ÉTAPE DU PROCESSUS D'APPROPRIATION PAR LE PROPRIÉTAIREDIRIGEANT ${ }^{5}$

\begin{tabular}{llll}
\hline $\begin{array}{l}\text { Type de conception } \\
\text { du SCdG }\end{array}$ & $\begin{array}{l}\text { Étape 1 } \\
\text { Adoption }\end{array}$ & $\begin{array}{l}\text { Étape 2 } \\
\text { Utilisation } \\
\text { contextualisée }\end{array}$ & $\begin{array}{l}\text { Étape 3 } \\
\text { Interaction }\end{array}$ \\
\hline Délégation & D G J & L & \\
\hline Externalisation & S V & B C M R W & P \\
\hline Autonomie & & & F K N \\
\hline
\end{tabular}

4 La présente recherche a pour objectif d'étudier des p.-dir. ayant sollicité un concepteur ; toutefois, la comparaison avec des p.-dir. autonomes est riche d'enseignements et ce groupe constitue un échantillon de référence.

5 L'identification des p.-dir. a été réalisée par une consonne, soit initiale du nom, soit incluse dans le nom, et constitue un moyen mnémotechnique pour l'auteur. 
Lorsque le processus s'est arrêté à l'étape 1, majoritairement la situation est celle de la délégation au métreur. Lorsque le processus d'appropriation est stoppé à l'étape 2 , très généralement la situation est celle de l'externalisation à l'expert-comptable. Lorsque l'étape 3 est atteinte, même si elle est parfois loin d'être terminée, le p.-dir. n’avait ni délégué ni externalisé la conception du SCdG, il s'agit de la conception en autonomie.

Les commentaires concernant chacune des trois situations de conception sont issus des constats lors des visites et des entretiens avec les p.-dir., illustrés de comptes rendus fidèles.

Lors de la délégation, le métreur construit un SCdG en mobilisant les connaissances qu’il met en œuvre le plus aisément et le plus souvent, celles qui lui sont le plus familières. Cette divergence, entre le métreur et le p.-dir., concernant les centres d'intérêt, crée une forte réticence des p.-dir. à s’approprier le travail élaboré par le métreur : «Seul un volet du CdG était couvert: il a mis en avant l'aspect marketing et la satisfaction des clients. Or il me fallait une vision globale de la situation et non un aspect parcellaire. » (P.-dir. D)

Par conséquent, ce p.-dir. considère qu'aucun SCdG n'est implémenté dans l'entreprise. Le P.-dir. G souhaitait un SCdG de pilotage ; or, le SCdG contient exclusivement des indicateurs physiques : "J'ai obtenu un $C d G$ qui se focalise sur la production, alors que je voyais un contrôle tactique, voire stratégique. »(P.-dir. G)

Le SCdG réalisé par le métreur de l'entreprise du P.-dir. J est orienté vers un aspect prescriptif, en raison de l'utilisation de références sectorielles, ainsi que de normes : «Sans vouloir le vexer, nous n'étions pas sur la même longueur d'onde. » (P.-dir. J)

Dans le cadre de l'externalisation auprès de l'expert-comptable, les p.-dir. n'ont pas réussi à venir totalement à la rencontre de la proposition de SCdG et à l'intégrer dans le processus de prise de décision. Certains p.-dir. considèrent que les conseils de l'expert-comptable sont identiques pour toutes les PE : "Je ne suis pas dupe qu'il [l'expert-comptable] donne les mêmes conseils à tous ses clients!»(P.-dir. W). La globalité de la perception de la PE par l'expert-comptable le conduit à proposer des solutions jugées, par les p.-dir., trop " clé en main» (P.-dir. C). Or, les entreprises ne sont pas toutes similaires, elles se destinent à un avenir différencié, car propre à la volonté de chaque p.-dir. : «Le CdG qu'il m'a proposé pourrait aussi bien convenir à une entreprise de n'importe quel secteur d'activité, avec n'importe quelle mise en perspective de l'avenir de l'entreprise. » (P.-dir. R)

Au demeurant, les p.-dir. n'ont pas clairement pris conscience de leur responsabilité quant à cet échec, au niveau de la communication : «On s'apprécie mutuellement avec l'expert-comptable, mais là, je pense qu'il n'a pas su, ou pas pris le temps, de vraiment comprendre ce que j'attendais de lui.» (P.-dir. B)

Il convient de noter des cas marginaux parmi des p.-dir. ayant eu recours à l'externalisation. Les P.-dir. S et V avaient initialement une vision trop floue et sans objectif précis du SCdG qu'ils souhaitaient implémenter. De plus, une collaboration de mauvaise qualité avec l'expert-comptable a conduit, d'une part, à l'abandon du projet de SCdG dans sa globalité, d'autre part, à une dégradation de leur relation. À l'inverse, le P.-dir. P n'a pas rencontré de difficulté à s'approprier le travail de l'expert-comptable avec qui il entretient une relation de confiance de longue date. 
Lorsque le p.-dir. décide de mettre seul en place le CdG, il en a conçu mentalement les objectifs. L'orientation du SCdG correspond donc exactement à ses attentes. Le processus d'appropriation peut alors être assimilé à une phase de réorganisation qui s'étendra dans le temps. Le P.-dir. N sait que quelques lacunes subsistent dans le SCdG qu’il a implémenté : « J’ai pas mal retravaillé sur mes propres tableaux, ratios, etc., et je continue d'ailleurs de temps à autre. Je sais que cela pourrait encore être amélioré. » (P.-dir. N)

Parfois plusieurs modifications ont été nécessaires pour parvenir au résultat attendu, l'anticipation mentale du choix des outils s'avérant quelquefois décevante après un court usage. Le point est mis sur l'impérative nécessité d'un test grandeur nature d'un tel SCdG, pendant quelque temps. Si l'amélioration des outils du CdG est toujours d'actualité, elle ne semble pas présenter un caractère d'urgence, ni un impératif, mais au contraire un projet à long terme : "Un tel système est toujours perfectible, je m'y emploie, mais c'est chronophage. Mais je sais exactement ce vers quoi je veux tendre. » (P.-dir. K)

La solide formation en gestion de ces trois p.-dir. autonomes est à remarquer, spécialement en termes d'homogénéité (les trois au niveau universitaire), contrairement à la diversité des types et niveaux d'études présentée par les p.-dir. des deux autres catégories.

\subsection{Conceptualisation des résultats : un lien entre le concepteur choisi et l'étape du processus d'appropriation par le propriétaire-dirigeant}

Il est possible de repérer l'étape à laquelle le processus d'appropriation s'est arrêté. Il apparaît assez nettement que, lorsque les p.-dir. ont délégué, le processus d'appropriation est arrêté à la première étape. Quand les p.-dir. ont externalisé, le processus est majoritairement interrompu à la deuxième étape. La troisième étape est en cours de réalisation par les p.-dir. qui ont construit leur SCdG en autonomie, comme le montre ce tableau conceptuel (Tableau 3). Cette étape semble pouvoir sans cesse accueillir de nouvelles modifications constructives du SCdG, par le biais des outils « maison ».

Tableau 3. Analyse Conceptuelle du processus D’appropriation Étape Par Étape

\begin{tabular}{llll}
\cline { 2 - 4 } & $\begin{array}{l}\text { Étape 1 } \\
\text { Adoption }\end{array}$ & $\begin{array}{l}\text { Étape 2 } \\
\text { Utilisation } \\
\text { contextualisée }\end{array}$ & $\begin{array}{l}\text { Étape 3 } \\
\text { Interaction }\end{array}$ \\
\hline Situation & Délégation & Externalisation & $\begin{array}{l}\text { Autonomie du p.-dir. } \\
\text { (création autour des } \\
\text { outils de CdG) }\end{array}$ \\
\hline $\begin{array}{l}\text { Frein principal } \\
\text { justifié par les p.-dir. }\end{array}$ & $\begin{array}{l}\text { Le SCdG est trop axé } \\
\text { sur un point précis } \\
\text { du fonctionnement de } \\
\text { l'entreprise qui n'est } \\
\text { pas pertinent pour le } \\
\text { p.-dir. }\end{array}$ & $\begin{array}{l}\text { Le SCdG ne répond } \\
\text { pas suffisamment } \\
\text { aux attentes et aux } \\
\text { objectifs du p.-dir., }\end{array}$ & \\
& $\begin{array}{l}\text { à ses valeurs et à } \\
\text { la culture de son } \\
\text { entrepris }\end{array}$ & \\
\hline
\end{tabular}




\begin{tabular}{|c|c|c|c|}
\hline & $\begin{array}{l}\text { Étape } 1 \\
\text { Adoption }\end{array}$ & $\begin{array}{l}\text { Étape } 2 \\
\text { Utilisation } \\
\text { contextualisée }\end{array}$ & $\begin{array}{l}\text { Étape } 3 \\
\text { Interaction }\end{array}$ \\
\hline $\begin{array}{l}\text { Raisons avancées } \\
\text { à l'existence d'un } \\
\text { obstacle dans le } \\
\text { SCdG proposé }\end{array}$ & $\begin{array}{l}\text { Confrontation de } \\
\text { compétences trop } \\
\text { spécifiques du métreur } \\
\text { à l'exigence de } \\
\text { polyvalence du p.-dir. } \\
\text { de PE }\end{array}$ & $\begin{array}{l}\text { Lacunes concernant } \\
\text { les échanges avec } \\
\text { l'expert-comptable au } \\
\text { sujet des aspirations } \\
\text { du p.-dir. }\end{array}$ & \\
\hline
\end{tabular}

La conceptualisation, concernant chacune des trois étapes, est maintenant présentée et analysée de manière détaillée.

\subsubsection{DÉlÉGATION : UN SYSTÈME DE CONTRÔLE DE GESTION SPÉCIFIQUE À UN DOMAINE TROP DÉLIMITÉ}

L'arrêt du processus d'appropriation dès la première étape semble assez spécifique à la situation de délégation. C'est dans ce cadre que l'incidence du principal capital de compétences du concepteur est plus spécialement ressentie. En dépit de l'éventail de compétences des métreurs, il semble que le biais se situe au niveau du domaine mis en valeur à travers le SCdG. Lorsque le p.-dir. recourt aux compétences d'un subordonné, le SCdG risque d'être orienté vers le domaine d'expertise du salarié concerné, et ainsi d'être en décalage par rapport aux attentes du p.-dir.

En effet, le métreur maîtrise pleinement le domaine très varié des calculs. Les objectifs répondent à des tâches bien précises, par exemple : optimiser les quantités de matière première, calculer au plus juste les besoins en quincaillerie (visserie, ferrure, pièces d'assemblage, etc.), minimiser des coûts de fabrication, démontrer au client le bien-fondé du prix fixé dans un devis, voire orienter le client vers une fabrication plus économique. L'estimation du temps nécessaire à une fabrication se fait par similitude avec d'autres chantiers, puisque nous sommes dans le domaine du sur-mesure, où chaque fabrication est spécifique. Cependant, au niveau du contrôle, seuls les calculs d'écart entre le prévisionnel et le réalisé en fabrication font vraiment partie de ses tâches au quotidien.

Le métreur met parfois en œuvre des connaissances techniques issues de fonctions antérieures dans d'autres entreprises, éventuellement d'un secteur d'activité différent - voire éloigné - du domaine de l'agencement.

Au demeurant, ses compétences, aussi variées qu'elles soient, sont confrontées à la polyvalence des activités du p.-dir. de PE et à la multiplicité des domaines de prise de décision.

\subsubsection{EXTERNALISATION : UN SYSTÈME DE CONTRÔLE DE GESTION TROP GÉNÉRIQUE À TOUS TYPES D'ENTREPRISE}

Le regard que porte l'expert-comptable concepteur sur l'organisation n'est pas assez individualisé sur cette entreprise précisément : le SCdG est jugé trop générique par le p.-dir. Il ne correspond pas à ses aspirations; de ce fait, le processus d'appropriation est en danger. En 
effet, devant la diversité des domaines d'activité des PE, il est compréhensible que l'expertcomptable peine à saisir toutes les nuances de chaque secteur d'activité, de chaque génération d'entreprise et surtout de chaque mode de gestion du p.-dir. Les p.-dir. retrouvent peu leurs attentes, en termes de mise sous contrôle, dans le système proposé par l'expert-comptable, mais surtout, ils ne se retrouvent pas eux-mêmes : leur vision de l'avenir et leurs projets ne sont pas suffisamment pris en compte par l'expert-comptable. Par exemple, les attentes, en termes de CdG, des p.-dir. qui ne souhaitent pas développer l'entreprise, se heurtent à des indicateurs de recherche - et même d'évaluation - de la croissance.

Au demeurant, les p.-dir. sont peu explicites et prolixes, concernant leur définition de l'avenir, auprès de leur expert-comptable. Ils semblent penser que leur politique d'entreprise " tombe sous le sens » (P.-dir. M), sans avoir à être précisée. Néanmoins, ils n’envisagent pas porter la responsabilité de l'arrêt du processus d'appropriation du SCdG lors de l'étape 2. L'étape de contextualisation ne fonctionne pas pour tous les couples « expert-comptable/p.dir. de PE ».

\subsubsection{AUTONOMIE : L’AMÉLIORATION CONTINUE DU SYSTÈME DE CONTRÔLE DE GESTION}

La création d'outils «maison » (Solle et Rouby, 2003) par réagencement des outils existants correspond à la phase maximale de l'appropriation. L'apprentissage vécu par le p.-dir., lors de la conception du SCdG, favorise le développement des connaissances et de la capacité à explorer tant le fonctionnement de l'entreprise que les outils de CdG. La troisième phase de l'appropriation est donc une période non limitée dans le temps.

Ainsi, lorsque le p.-dir. a la possibilité de construire lui-même son SCdG, le bénéfice va audelà de l'instrumentation. Ce sont ses capacités de structuration, d'apprentissage, d'exploration qui sont stimulées.

À l'inverse, malgré le souhait du p.-dir. - justifié en termes de faible disponibilité et de compétences modérées - de déléguer ou d'externaliser à un concepteur, ces deux situations ne sont pas favorables à la mise en œuvre d'un SCdG si elles ne sont pas abordées avec précaution quant à la relation entre le p.-dir. et le concepteur. Il est alors judicieux de s'interroger sur la légitimité du concepteur en fonction de ses relations avec le p.-dir. et sur les freins à l'action que l'intervention de cet acteur dans le SCdG peut induire (Bernard et Culié, 2016).

\section{DISCUSSION AUTOUR DES APPORTS DE LA PRÉSENTE RECHERCHE}

Outre l'apport d'un modèle de synthèse de la littérature par le biais de la grille de lecture du processus d'appropriation, cette étude délivre à la fois des apports théoriques et des apports managériaux. 


\subsection{Apports théoriques}

Dans le cadre des travaux de Piaget (1973), le passage de la phase d'assimilation à celle de l'accommodation exige l'acceptation par l'acteur de faire évoluer - voire de modifier - ses croyances et ses valeurs. Transposé à notre problématique, ce franchissement pour le p.dir. correspond à avoir suffisamment intégré le SCdG proposé par le concepteur - dans ses réflexions comme dans son quotidien - pour pouvoir le réinterpréter.

Plusieurs freins peuvent restreindre le passage de la phase d'assimilation à la phase de l'accommodation par le p.-dir. ; la revue de la littérature a permis de les identifier et ils sont justifiés par l'étude empirique. Si le concepteur impose son approche du CdG par le biais d'une problématique spécifique, propre à l'expérience professionnelle du concepteur, elle ne correspond pas à la polyvalence du p.-dir. On comprend alors que le p.-dir. ne perçoive dans le SCdG proposé qu'une fraction, plus ou moins vitale, des problèmes qu'il rencontre. Le mécanisme d'accommodation ne peut bien sûr pas s'effectuer sur la base de cette vision parcellaire.

Les p.-dir. ont tendance à mettre en doute la juste perception de leurs difficultés par les conseillers, y compris par l'expert-comptable. Ils ont donc tendance à camper sur leur position, à ne pas questionner de nouveau le bien-fondé de leurs attentes initiales en termes de $\mathrm{CdG}$ et à ne pas modifier leurs schèmes de réflexion. Le concepteur, dans un contexte de communication insuffisante, construit le SCdG sur la base des hypothèses qu'il formule. Ainsi, ces conjectures risquent d'être trop personnelles, trop éloignées des aspirations du p.dir. Les liens nécessaires à l'accommodation, que le p.-dir. doit réaliser, entre la proposition de SCdG et de nouveaux schèmes cognitifs, ne se créeront pas. En effet, Cullière (2006, p. 175) conseille une situation où «le conseil met en jeu des savoirs détenus par les acteurs, davantage que des actions de soutien génériques et préformatées ». Le partage de la vision de l'avenir de l'entreprise étant absent, la mise sous contrôle de la stratégie ne peut pas être cohérente. En ce sens, Cullière (2006) fait référence aux travaux de Dalley et Hamilton (2000), " s'il apparaît que la transmission des savoirs entre en conflit avec le contexte de l'entreprise, les nouvelles informations seront rejetées pour ne jamais être transformées en connaissances ».

$\mathrm{Au}$ demeurant, sous un aspect théorique, d'autres points se révèlent beaucoup plus positifs et orientés dans le sens d'une transformation des schèmes de pensée. L'action structurante des outils de gestion facilite l'évolution de la réflexion par paliers ou concernant des thèmes précis. Structurer, organiser la réflexion permet aussi de la faire avancer. La richesse, liée aux divergences entre les organisations, mise en avant grâce à l'action du concepteur du SCdG constitue un terrain propice à l'évolution des schèmes de pensée du p.-dir. Toute disparité, bien acceptée, est constructive en termes d'ouverture d'esprit. L'existence de désaccords, entre le concepteur et l'utilisateur du SCdG, crée un déséquilibre bénéfique à l'évolution des croyances du p.-dir. Une situation d'instabilité cognitive génère un besoin de rééquilibration, qui peut permettre d'orienter la réflexion vers une autre voie, que ce soit celle proposée par le concepteur du SCdG ou - de manière encore plus favorable - une voie totalement nouvelle, née de cette confrontation.

Les facteurs facilitateurs de la part du concepteur, en termes de compétences relationnelles, d'empathie et de pédagogie, jouent un rôle non négligeable dans la rééquilibration et incitent à la transformation des schèmes de pensée de l'utilisateur. Lappropriation des outils par le 
p.-dir. s'effectue d'autant plus facilement que la relation entre le p.-dir. et le concepteur est de bonne qualité, car le fait de se sentir épaulé est un facilitateur de l'action d'appropriation du SCdG proposé. En effet, Barlette et Jaouen (2014, p. 634) montrent que «le processus de production d'un conseil est souvent associé à un processus d'échange qui implique un apprentissage de la part des deux parties impliquées ». En ce sens, un rôle de traducteur - selon la théorie de la traduction (Callon, 1986, dans Nobre et Zawadzki, 2015) - peut être attribué au concepteur dans sa fonction pédagogue.

\subsection{Apports managériaux}

Des préconisations se dégagent de cette approche du processus d’appropriation. En situation de délégation, une communication renforcée pourrait éviter une délégation à l'aveugle, simplement fondée sur le ressenti de sécurité que représentent les compétences techniques spécifiques du métreur. Un échange clair, autour des objectifs de stratégie globale et du contrôle stratégique souhaité, pourrait permettre un travail plus collaboratif.

En situation d'externalisation, la nécessité pour les p.-dir. de communiquer, auprès de l'expert-comptable, sur leur vision de l'avenir de l'entreprise est impérative. Généralement, cette vision est très peu formalisée et difficile à exprimer. C'est ce double effort qui est nécessaire dans la situation d'externalisation. D'une part, avoir une vision la plus précise possible de l'avenir de l'entreprise, afin de pouvoir l'expliciter; d'autre part, concevoir que leur réflexion personnelle doit être communiquée. En effet, les p.-dir. sont peu précis au sujet de l'avenir de l'entreprise comme de leur devenir personnel. Ils n'accordent pas suffisamment d'importance aux précisions concernant leurs propres attentes de mise sous contrôle. Les p.-dir. doivent prendre conscience du besoin de communiquer sur leur vision de l'avenir, même si cette vision est très peu formalisée et difficile à exprimer.

Ainsi, les points-clés des échanges avec le métreur porteraient sur le besoin d'une vision globale du fonctionnement de l'entreprise, avec des points de détail ciblés sur la gestion quotidienne. Ce même échange avec l'expert-comptable devrait porter sur la définition de la vision pour l'entreprise, des objectifs tant de l'entreprise que du CdG, des attentes spécifiques, du design du SCdG, des éléments non souhaités.

\section{CONCLUSION}

L'objectif de cette étude était de comprendre l'incidence de la situation d'externalisation ou de délégation de la conception du SCdG sur le processus d'appropriation par le p.-dir., à l'issue duquel le SCdG proposé devient opérationnel et constitue une aide à la prise de décision. Des SCdG sont souvent réduits à néant après quelques mois, car le p.-dir., dans son rôle de contrôleur de gestion, ne parvient pas à trouver de réponses à ses attentes et aux besoins de l'entreprise tels qu'il les conçoit, et il abandonne. Des préconisations, fruit du croisement entre la revue de la littérature concernant les freins à l'appropriation et les discours des p.-dir., concernent la qualité de la communication du p.-dir. quant à ses attentes et les besoins de l'entreprise telle qu'il les perçoit. 
Une limite de cette étude concerne le rôle de la subjectivité, inévitable, car inhérente à la nature humaine tant du répondant que du chercheur. Or, le besoin de cette étude en termes de collecte d'informations concerne des concepts immatériels. Ainsi, cette recherche est confrontée à l'obstacle de l'expression par les p.-dir. et à des problèmes de repérage, par le chercheur, des informations dans le discours des p.-dir. Ces risques - tels qu'un risque de justification a posteriori par le p.-dir. - ont été anticipés, mais n’ont, pour autant, pas pu être totalement éliminés.

Cette étude porte sur les facteurs de contingence individuels et comportementaux, aussi, convient-il d'introduire dans les limites de cette recherche les facteurs de contingence organisationnels et environnementaux.

La branche d'activité choisie - la fabrication d'agencement sur mesure, riche de fortes traditions issues du secteur du bois - incarne un secteur spécialement peu enclin à communiquer sur ses pratiques de gestion : attentes du SCdG, actions à privilégier, stratégie...

Si chaque étude de cas est très approfondie, le nombre de cas étudiés reste néanmoins limité. Il serait scientifiquement utile d'accroître la taille de l'échantillon : de plus nombreuses investigations permettraient de conforter les résultats obtenus et d'affiner les préconisations. En effet, s'il est possible d'étudier chaque type de situation - délégation, externalisation ou autonomie - la taille de l'échantillon n'autorise pas à multiplier les cas concernant chaque situation.

L'une des conditions techniques favorisant l'appropriation, par le p.-dir., du SCdG est l'amélioration du système d'information interne, comme externe. En effet, ils sont généralement insuffisamment développés en PE, ce qui pénalise le SCdG. Des conseils concernant la nécessité du renforcement du système d'information pour favoriser l'utilisation du SCdG seraient souhaitables. Cette préconisation demanderait une exploration spécifique ultérieure.

Enfin, en termes de mise en perspective, Thomas et Schwenck (1983) suggèrent une position encore trop rarement rencontrée en entreprise, bien que novatrice : l'écart entre les attentes du p.-dir. et la proposition de SCdG devrait être considéré comme une opportunité à saisir. Tout un travail de prospective, avec le p.-dir., serait alors à envisager, et corroborerait la vision de l'appropriation par Grimand (2006, p. 20) comme « une opportunité de repenser les rapports entre conception et usage ". 


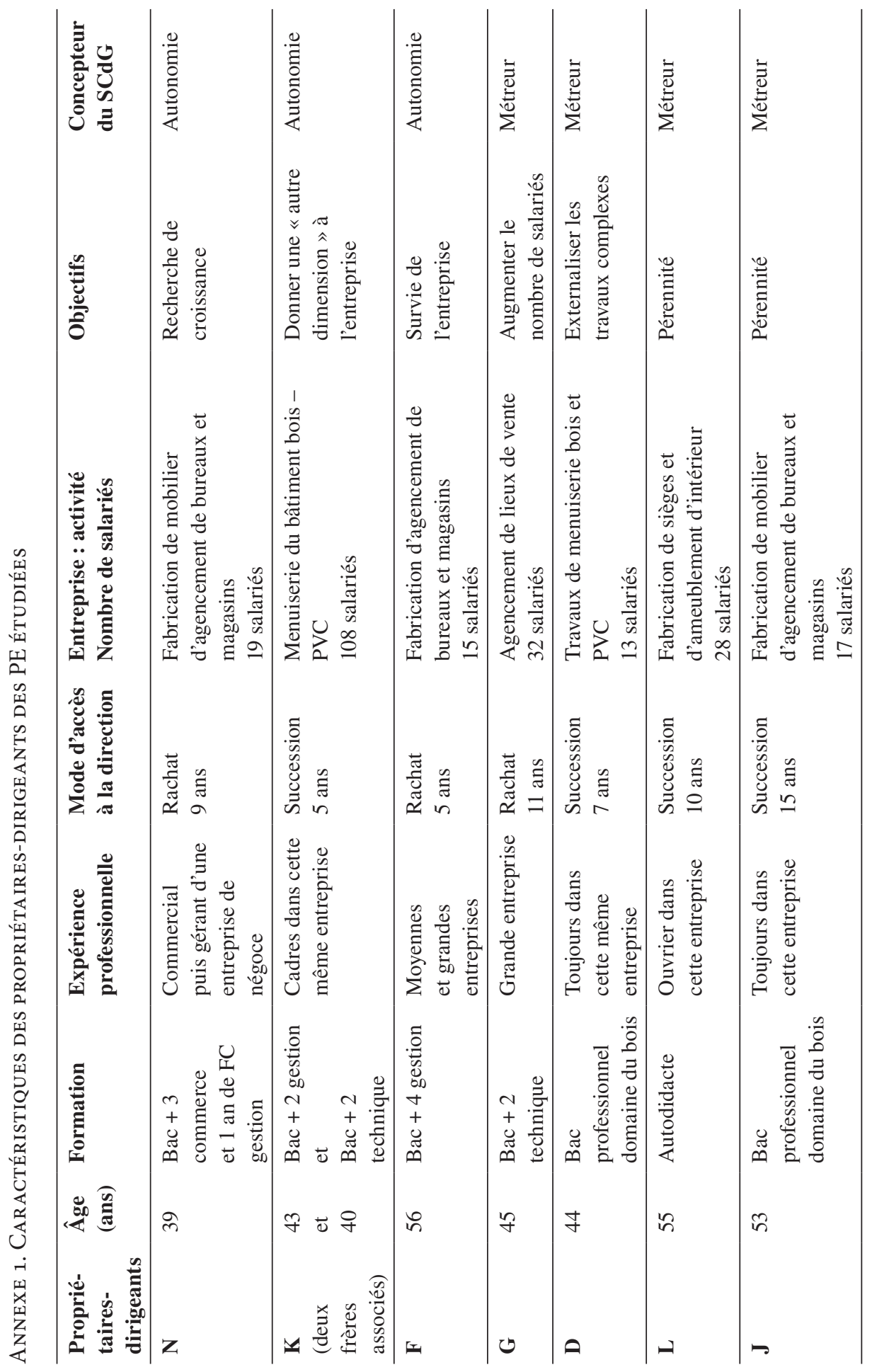




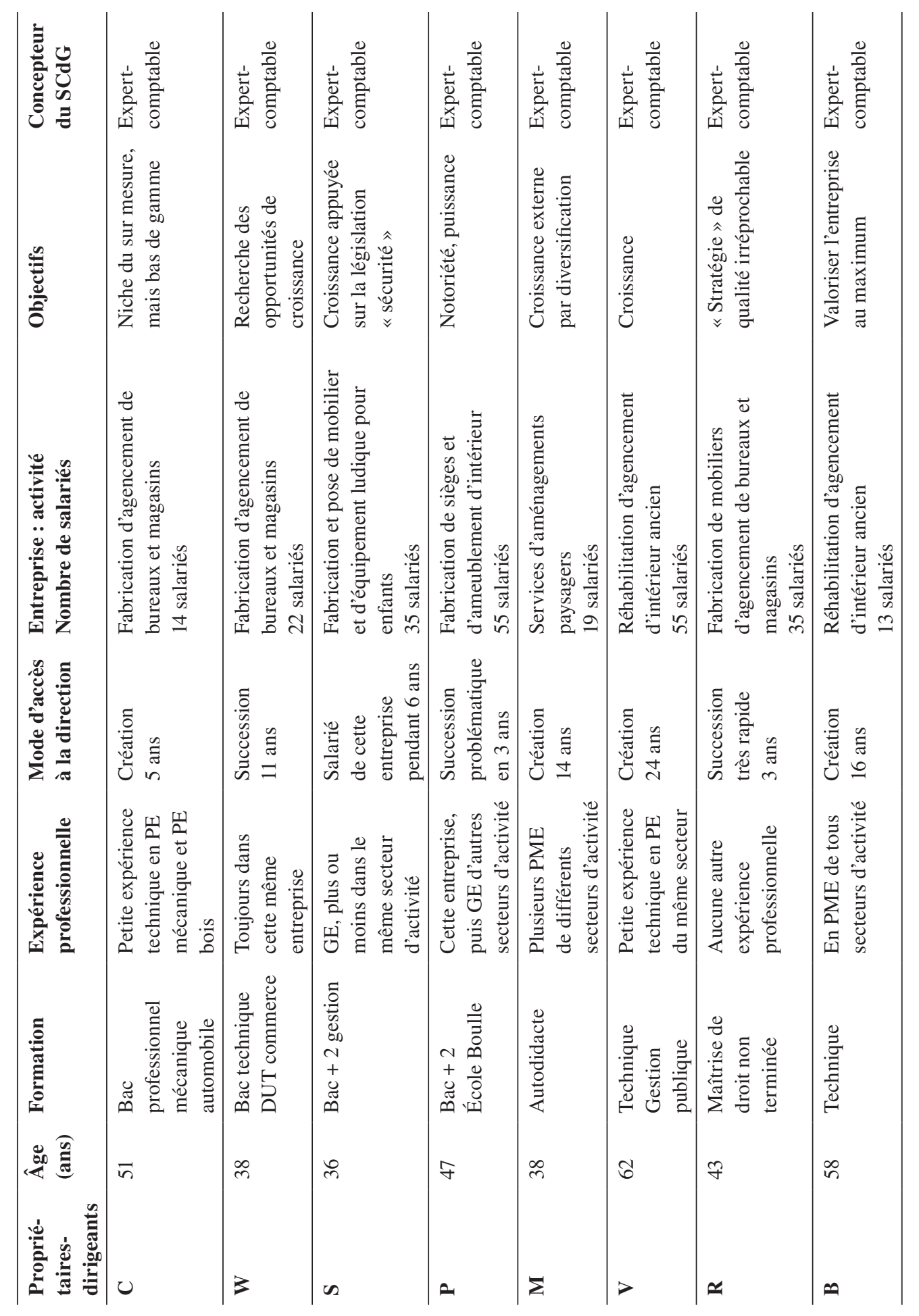




\section{RÉFÉRENCES}

Abdallah C. (2007). Production et appropriation du discours stratégique dans une organisation artistique. Revue française de gestion, 33(174), 62-76.

Barlette, Y. et Jaouen, A. (2014). La relation entre le dirigeant et son expert-comptable en matière de gestion des informations. Dans G. Lecointre (dir.), Le Grand Livre de l'économie PME (p. 629-640). Paris, Gualino Éditions.

Bennett, R.J. et Robson, P.J.A. (1999). The use of external business advice by SMEs in Britain. Entrepreneurship \& Regional development, 11, 155-180.

Bennett, R.J. et Robson, P.J.A. (2005). The advisor-SME client relationship : impact, satisfaction and commitment. Small Business Economics, 25(3), 255-271.

BERNARD, O. et Culié, J.-D. (2016). Le dirigeant de petite entreprise sollicite une aide pour la création d'un système de contrôle de gestion : la légitimité du concepteur. Revue management \& avenir, (86), $15-34$.

Bessire, D., Brillet, F., Grimand, F. et Meric, J. (2012). De l'adoption à l'appropriation : une exploration croisée des liens entre outils de gestion et organisation. Revue management \& avenir, (54), introduction.

Blanchet, A. et Gotman, A. (2005). L'enquête et ses méthodes : l'entretien. Paris, Armand Colin.

Bonneveux, E. et CAlmé, I. (2010). Le processus d'appropriation d'une démarche de RSE par des dirigeants de petites entreprises. Dans les Actes du $10^{e}$ Congrès CIFEPME. Bordeaux, Université Bordeaux IV, 27 octobre.

Bonneveux, E., CAlmé, I. et Soparnot, R. (2016). Le processus d'appropriation d'une démarche RSE par les dirigeants de PME. Revue internationale PME, 29(3/4), 241-268.

Bourguignon, A. (2004). Performance, management and management control : evaluated managers' point of view. European Accounting Review, 13(4), 659-687.

Brasseur, M. et Buisson, M.-L. (2008). La fin du dilemme universalisme vs contingence ? La transférabilité des pratiques de gestion, une approche par le concept de légitimité. Dans les Actes du $19^{e}$ Congrès AGRH. Dakar, novembre.

Breton, P. et Proulx, S. (2002). L'explosion de la communication à l'aube du XXI ${ }^{e}$ siècle. Paris, Éditions La Découverte/Montréal, Boréal.

Chapellier, P., Mohammed, A. et Teller, R. (2013). Le système d'information comptable des dirigeants de PME syriennes : complexité et contingence. Revue management \& avenir, (65), 48-72.

Charreire, S. (2002). Chris Argyris. Apprentissage organisationnel, actionnabilité des connaissances et vision pragmatique. Dans S. Charreire et I. Huault (dir.), Les Grands Auteurs en management, Cormelles-le-Royal, Éditions EMS.

Cullière, O. (2006). Appropriation du conseil externe et légitimité du prescripteur. Dans A. Grimand (dir.), L’Appropriation des outils de gestion (p. 175-198). Saint-Étienne, Publications de l'Université Saint-Étienne.

Dalley, J. et Hamilton, B. (2000). Knowledge, context and learning in the small business. International Small Business Journal, 18(3), 51-59. 
Dangereux, K., Chapellier, P. et Villesèque-Dubus, F. (2017). Adapter les outils de contrôle aux contextes et aux acteurs dans les PME : le cas exploratoire du tableau de bord achat d'un dirigeant. Revue internationale PME, 30(1), 27-56.

DAVID, A. (1996). Structure et dynamique des innovations managériales. $5^{e}$ conférence de l'AIMS. Lille, France, 13 au 15 mai.

Davila, A. et Foster, G. (2005). Management accounting systems adoption decisions : evidence and performance implications from early-stage/startup companies. The Accounting Review, 80(4), 10391068.

DeLone, W.H. et McLean, E.R. (2003). Model of information systems success : a ten-year update. Journal of Management Information Systems, 19(4), 9-30.

De Vaujany, F.X. (2005). De la conception à l'usage: vers un management de l'appropriation des outils de gestion. Cormelles-le-Royal, Éditions EMS.

Dyer, L.M. et Ross, C.A. (2007). Advising the small business client. International Small Business Journal, 25(2), 130-151.

Gable, G.G. et Chin, W.W. (2001). Client versus consultant influence on client involvement in computer system selection projects. The $22^{\text {nd }}$ International Conference on Information Systems. NouvelleOrléans, États-Unis, décembre.

Germain, C. (2006). Le pilotage de la performance dans les PME en France : une comparaison des pratiques de tableaux de bord des organisations familiales et des filiales. Revue internationale PME, 19(1), 69-94.

Gervais, M. (2005). Contrôle de Gestion (8 édition). Paris, Economica.

GiBB, A.A. (2000). SME policy, academic research and the growth of ignorance, mytical concepts, myths, assumptions, rituals and confusions. International Small Business Journal, 18(3), 13-35.

Grimand, A. (2006). Une analyse des blocages à l'appropriation des outils de gestion des ressources humaines : l'exemple du management des compétences. Préactis.

Grimand, A. (2012). L'appropriation des outils de gestion et ses effets sur les dynamiques organisationnelles : le cas du déploiement d'un référentiel des emplois et des compétences ». Revue management \& avenir, (54), 237-257.

Grimand, A. et Bachelard, O. (2005). La dynamique de la formation et de l'appropriation des connaissances. Dans F.-X. de Vaujany (dir.), De la conception à l'usage (p. 149 à 198). Cormelles-leRoyal, Éditions EMS.

Hussenot, A. (2008). Between structuration and translation : an approach of ICT appropriation. Journal of Organizational Change Management, 21(3), 335-347.

Julien, P.-A. (2005). Les PME : bilan et perspectives (3e édition). Paris, Economica.

Mallet, C. (2006). Innovation et mesure de l'appropriation des outils de gestion : proposition d'une démarche de construction d'un tableau de bord. Colloque "en route vers Lisbonne " du Centre de recherche public H. Tudor et de l'Observatoire de la compétitivité luxembourgeois. Luxembourg, France, 9 novembre.

Marriott, N. et Marriott, P. (2000). Professional accountants and the development of a management accounting service for the small firm : barriers and possibilities. Management Accounting Research, 11, 475-492. 
Massard, N. (2009). Revisiter la notion d'appropriation : pour une application au cas des ERP. $14^{e} \mathrm{col}-$ loque de l'Association informatique et management. Marrakech, Maroc, 10 au 12 juin.

Mintzberg, H., Ahlstrand, B. et Lampel, J. (2005). Safari en pays stratégique, l'exploration des grands courants de la pensée stratégique. Paris, Pearson Education France.

Moisdon, J.C. (1997). Du mode d'existence des outils de gestion. Paris, Éditions Seli Arslan.

MoLE, K. (2002). Business adviser's impact on SMEs : an agency theory approach. International Small Business Journal, 20(2), 139-162.

Nieto-Bru, G. (2009). L’appropriation des outils de gestion du risque dans les projets : le cas du Crédit Agricole (thèse de doctorat en sciences de gestion). Orléans, Université d'Orléans.

Nobre, T. et Zawadzky, C. (2014). Le contrôle de gestion dans la PME : mythe ou réalité. Dans M. Bollecker et G. Naro (dir.), Le contrôle de gestion aujourd'hui (p. 243-256). Paris, Éditions Vuibert.

Nobre, T. et Zawadzky, C. (2015). Analyse par la théorie de la traduction de l'abandon et du détournement d'outils lors de l'introduction d'un contrôle de gestion en PME. Finance Contrôle Stratégie, 18(1). Récupéré le 28 mai 2015 sur le site : http://journals.openedition.org/fcs/1574.

Perez, M., Chalayer-Rouchon, S. et Teyssier, C. (2005). Une approche socio-politique et psychocognitive des outils de gestion comptables et financiers. $26^{e}$ congrès de l'Association française de comptabilité. Lille, France, 11 au 13 mai.

Persson-Gehin, S. et Dokaj-IvanaJ, V. (2002). L’influence des valeurs du dirigeant sur la stratégie de croissance de la PME. Cahier de recherche Nancy 2, (95.02).

PiAget, J. (1973). Biologie et connaissance : essai sur les relations entre les régulations organiques et les processus cognitifs. Paris, Gallimard.

Proulx, S. (2002). Trajectoires d'usage des technologies de communication : les formes d'appropriation d'une culture numérique comme enjeu d'une société du savoir. Annales des Télécommunications, 57(3/4), 180-189.

Proulx, S. (2005). Penser les usages des TIC aujourd'hui : enjeux, modèles, tendances. Dans L. Vieira et N. Pinede (dir.), Enjeux et usages des TIC : aspects sociaux et culturels (p. 7-20). Bordeaux, Presses universitaires de Bordeaux.

Schmitt, C., Julien, P.-A. et Lachance, R. (2002). Pour une lecture des problèmes complexes en PME : approche conceptuelle et expérimentation. Revue internationale PME, 15(2), 35-62.

Solle, G. et Rouby, E. (2003). De la conception des innovations managériales en contrôle de gestion : quelles propositions ? Comptabilité Contrôle Audit, (numéro spécial), 147-168.

Stumpf, S. et Longman, R.A. (2000). The ultimate consultant : building long-term, exceptional value client relationships. Career Development International, (5/3), 124-134.

Thomas, H. et Schwenck, C. (1983). Problem formulation and the consultant-client relationship. Interfaces, 13(5), 25-34.

WANG, E.T. et ChEN, J.H. (2006). Effects of internal support and consultant quality on the consulting process and ERP system quality. Decision Support Systems, 42(2), 1029-1041.

YIN, R.K. (1989). Case study research. Design and methods. Thousand Oaks, Sage Publications. 